\title{
Kuwaiti Mothers' Perception of Their Child's Body Mass Index and Their Belief in Early Childhood Dietary Advice
}

\author{
Ghanima Alabdullah ${ }^{1}$, Joan Vaccaro ${ }^{1 *}$, Deborah Abel ${ }^{1}$, Lemia Habib ${ }^{2}$ and Fatma Huffman ${ }^{1}$ \\ ${ }^{1}$ Department of Dietetics and Nutrition, Florida International University, USA \\ ${ }^{2}$ Department Food Science and Nutrition, Kuwait University, Kuwait
}

Submission: March 06, 2018; Published: March 26, 2018

*Corresponding author: Joan A Vaccaro, Department of Dietetics and Nutrition, Florida International University, Robert R. Stempel College of Public Health and Social Work, 11200 SW, $8^{\text {th }}$ ST, AHC-5 Room 324, Miami, FL 33199, USA, Tel: (305) 348-0368; Fax: (305) 348-1996; Email: jvaccaro@fiu.edu

\begin{abstract}
Aim: The aim of this study was to assess Kuwaiti mothers' perception of their child's body weight and the child's actual age/sex body mass index. A secondary aim was to compare health beliefs for dietary advice with child's body weight.
\end{abstract}

Methods: This cross-sectional study involved dyads of Kuwaiti children aged 3-6 years and their mothers (N=67). Subjects were invited during summer 2014 from five summer camps in five different areas in Kuwait. Recruitment was by a flyer about the study in Arabic. Participants were children who gave assent and mothers who signed informed consent. Questionnaires were administered to assess health beliefs, history of obesity, professional and media dietary advice, and perception about their child's BMI.

Results: Forty-two (62.7\%) of the 67 children had a normal body weight; 14 (20.9\%) were underweight and $11(16.4 \%)$ were overweight or obese. Approximately half the mothers did not correctly perceive their child's body weight. Less than $5 \%$ of mothers with children in the overweight/obese category correctly classified their child, while over $15 \%$ were overweight/obese.

Conclusion: Interventions that educate Kuwaiti mothers to correctly assess their child's weight could serve as a strategy to reduce child obesity and correct under nutrition.

Keywords: Mother-child dyads; Child obesity; Dietary advice; Health beliefs; Body weight perception; Kuwait

Abbreviations: BMI: Body Mass Index for Sex/Age; IRB: Institutional Review Board; CDC: Center of Disease Control and Prevention

\section{Introduction}

Kuwait is a small county located on the coast of the Arabian Gulf where oil industry is the main source of the national income. The total population of Kuwait is 4.1 million, $70 \%$ of the population are expatriates, and 98\% are urbanized [1]. The harsh climate of Kuwait along with the development and availability of technology has led to sedentary lifestyles, which has contributed to the increase of the prevalence of obesity [2]. According to Kuwait Nutrition Surveillance System 5\% of male preschool children $(<5$ years) and $7 \%$ of female preschool children are obese [3].

Childhood obesity can affect the child quality of life by affecting not only the child's health but also the child's social and academic life [4-6]. In addition, the health consequences of childhood obesity not only affect their health in the present, it also affects their health in the long-term. Studies had shown that obesity during childhood, leads to obesity over the lifecycle $[5,6]$. Also, there is strong evidence on the association between childhood overweight and obesity with physical morbidity including diabetes and heart diseases during adulthood [5,7].

What the child eats can determine the child's weight. Caretakers, especially the mother, have major influence on the children's eating habits. If the mother misperceives her child's weight status, the child can be at risk of obesity [4,8-12]. The goal of having a society that is free from obese children starts with the parents [13]. Factors that can lead the mother to misperceive her child's weight status include: parent weight status, education level of the mother, child's gender, family history, and not knowing how much the child should weigh 


\section{Current Research in Diabetes \& Obesity Journal}

[8,12-14]. In addition, the image that the chubby child is a healthy child is very common in this culture [15].

According to a systematic review, $62.4 \%$ of the overweight children were incorrectly perceived by their parents as having normal weight [13]. In the United Arab Emirates, which has a similar demographic of socioeconomic status to Kuwait, one third of the parents misclassified their children's weight and $63.5 \%$ of the misclassifications were from the parents of overweight or obese children [16]. In Kuwait between 2003-2004, 73.1\% of obese children and $97 \%$ of overweight children were incorrectly perceived by their mothers [12]. During the following years, a new department of Food Science and Nutrition was opened in Kuwait University, which led to an increase in the number of nutritionists working on improving the nutritional knowledge of the Kuwaiti population. In addition, with the increased use of social media worldwide, nutritionists in Kuwait have been using social media to provide nutrition information. On the other hand, the lifestyle in Kuwait also changed to more sedentary lifestyle and the eating habits are adapting the western calories dense diet. Due to the change in environment in Kuwait over the past 10 years, the relationship between mothers' perception of their child's weight and the child's actual weight is not known.

The primary aim of this study was to assess the difference between the Kuwaiti mother's perception of her child's BMI and the child's actual measured BMI. The secondary aim was to determine if mothers classified weight more accurately for boy as compared to girls. A third aim of this study was to observe if the health belief that early weight management for their child was associated with correct perception of their child's body weight classification.

\section{Methods}

\section{Participants}

The study was approved by the Institutional Review Board (IRB) through Florida International University IRB Protocol Exemption \#: IRB-14-0134. All children assented and mothers signed informed consent. This was a cross sectional design of mother-child dyads conducted in summer 2014. The inclusion criteria specified that mothers and children were of Kuwait nationality and Kuwaiti children were ages 3-6 years. Exclusion criteria: Non-Kuwaiti mothers or children that are not in the age range of 3-6 years, wearing diapers, having physical disabilities, or children with any chronic disease or syndrome were excluded from the study. The participants were invited from 5 summer camps in 5 different areas in Kuwait: Al-Rawda, Al-Salam, AlSalmia, Al-Adan; and Meshref. The five participating summer camps are comparable; offering similar activities with the main focus of daycare and enjoyment. Activities include: play time, English language, math, and art and crafts and having participants at the same average socioeconomic status, boy and girls, and at the age between 3-6 years old, and were selected randomly from the expo of available summer camps for children in Kuwait. Measurements were taken at each camp. Mothers of non-Kuwaiti origin, children having physical disabilities, or children with any chronic disease or syndrome were excluded from the study.

Children were sent home with flyers explaining the study written in Arabic language, the main language of the country, and asked if they would like to participate in the study. Mothers who agreed to participate gave consent and to be included in the study, their child gave assent. Participants completed Arabic translated questionnaire that reflects their perception on her child BMI, demographics, and health beliefs and send it back with her child. Mothers' perception of their child's weight was based on the question: "How do you categorize the child's weight: underweight, normal, overweight, or obese?" The mothers' weight and height were self-report in $\mathrm{kg}$ and $\mathrm{cm}$. The mothers' BMI was calculated by dividing the weight in kilograms over the height in meter square $\left(\mathrm{kg} / \mathrm{m}^{2}\right)$. Mothers were classified as obese if their BMI was above 30, overweight if it was between 25.0-29.9, normal weight if it was between $18.5-24.9$, and underweight if it was below 18.5 [17]. Children's weight were measured in light clothing without shoes by regularly calibrated electronic scale placed in a hard, flat, and even surface. The children were asked to stand on the center of the scale with their feet slightly apart and not to touch anything, and their weight was recorded to the nearest $0.1 \mathrm{~kg}$. The children' height was measured without shoes or hair accessories with a stadiometer placed against the wall. They were asked to stand straight with their back against the wall and their head straight, and their height was recorded to the nearest $0.1 \mathrm{~cm}$. The children's BMI was calculated using the boys or girls BMI-for-age CDC Child Growth Standards [18]. The CDC chart for children and adolescents generates the sex and age specific BMI percentile. Adults were calculated using weight in $\mathrm{kg}$ and height in meters $\left(\mathrm{BMI}=\mathrm{kg} / \mathrm{m}^{2}\right)$ Using the CDC's standard for BMI classification, children and adults were classified as obese if their BMI was $\geq 95^{\text {th }}$ percentile, overweight if their BMI was between $\geq 85^{\text {th }}$ and $<95^{\text {th }}$, normal weight if their BMI was between $\geq 5^{\text {th }}$ and $<85^{\text {th }}$, and underweight if the BMI is $<5^{\text {th }}$ $[18,19]$.

\section{Statistical Analyses}

The sample size was calculated using the $G^{*}$ Power version 3.17. Two tailed paired t-test was used to compare the differences between the estimated BMI and the actual BMI. The $\alpha$ level was set to $5 \%(0.05)$, the power level to $95 \%(0.95)$, and medium effect size (0.50). As a result, a sample size equal to 54 was suggested. Data were collected using REDCap and analyzed by using Statistical Package for Social Sciences (SPSS) version 23. Cross-tabulations were run with the chi-squared test to assess the difference between Kuwaiti mothers' self-reported perception of their child's BMI and the actual measurement BMI, child's gender, and health belief for dietary intervention early in the child's life. Linear mixed model with dyads was conducted with mother's perception of their child's weight, child's sex, and the interaction of perception and sex. 


\section{Current Research in Diabetes \& Obesity Journal}

Results

A total of 100 pairs (100 children and their mothers) were invited to participate by sending children home with the study flyer, attached consent forms, and the questionnaire for their

Table 1: Characteristics of study subjects $(n=67)$. mothers to complete. The response rate was $71 \%$ and of the 71 pairs (Alresala summer camp $n=20$, Alyasmeen $n=12$, Altamayoz $n=14$, Victoria $n=12$, Joy camp $n=13$ ). The final sample size was 67 after applying the inclusion criteria; 4 were excluded because they are non-Kuwaitis.

\begin{tabular}{|c|c|c|}
\hline Demographic Variable & $\mathbf{N}$ & $\%$ \\
\hline \multicolumn{3}{|c|}{ Children's age (years) } \\
\hline 3 & 30 & 44.8 \\
\hline 4 & 30 & 44.8 \\
\hline 5 & 5 & 7.4 \\
\hline 6 & 2 & 3 \\
\hline \multicolumn{3}{|c|}{ Child's BMI percentile classification } \\
\hline Under weight & 14 & 20.9 \\
\hline Normal weight & 42 & 62.7 \\
\hline Overweight/obese & 11 & 16.4 \\
\hline \multicolumn{3}{|c|}{ Children's sex } \\
\hline Male & 36 & 53.7 \\
\hline Female & 31 & 46.3 \\
\hline \multicolumn{3}{|c|}{ Child's rank in the family } \\
\hline $1^{\text {st }}$ & 33 & 49.3 \\
\hline $2^{\text {nd }}$ & 19 & 28.4 \\
\hline $3^{\text {rd }}$ & 8 & 11.9 \\
\hline $4^{\text {th }} \geq$ & 7 & 10.4 \\
\hline \multicolumn{3}{|c|}{ Number of siblings } \\
\hline $1 \mathrm{~s}$ & 9 & 13.5 \\
\hline 2 & 24 & 35.8 \\
\hline 3 & 21 & 31.3 \\
\hline $4 \geq$ & 13 & 19.4 \\
\hline \multicolumn{3}{|c|}{ Mother's weight status } \\
\hline normal & 21 & 31.3 \\
\hline overweight & 26 & 38.8 \\
\hline obese & 6 & 9 \\
\hline didn't report & 14 & 20.9 \\
\hline \multicolumn{3}{|c|}{ Mother's age (y) } \\
\hline $20-29$ & 31 & 46.3 \\
\hline $30-39$ & 34 & 50.7 \\
\hline $40-49$ & 1 & 1.5 \\
\hline $50 \geq$ & 1 & 1.5 \\
\hline \multicolumn{3}{|c|}{ Mother's education level } \\
\hline at high school level or below & 2 & 97 \\
\hline above high school level & 65 & 3 \\
\hline \multicolumn{3}{|c|}{ Mother's working status } \\
\hline working & 60 & 89.6 \\
\hline housewife & 7 & 10.4 \\
\hline
\end{tabular}

The characteristics of study subjects are shown in (Table 1). Both under and overweight/obese appear to be issues in this sample since $20 \%$ were underweight and $16 \%$ were overweight/ obese. Out of the 67 mothers, 14 refused to report their perceived body weight classification $(20.8 \%)$; of the remaining 53,21 reported their weight to be 'normal' (39.6\%); 26 reported 


\section{Current Research in Diabetes \& Obesity Journal}

themselves to be 'overweight' (49.0\%) and 6 reported themselves to be 'obese' (11.3\%). Forty-two (62.7\%) of the 67 children had a normal body weight; 14 (20.9\%) were underweight and 11 (16.4\%) were overweight or obese. Nearly $30 \%(n=20,29.9 \%)$ of mothers believed that children did not need early childhood weight management; whereas approximately 70\% $\quad n=47$, $70.1 \%$ ) believed that early childhood weight management was essential. Approximately half of mothers received dietary advice online $(n=35,52.2 \%)$. Over $40 \%(n=28,44.0 \%)$ did not correctly classify their child's weight. Mother's perception of their child's weight was not significantly associated with the mother's occupational status, number of children, sex of the child, or education level.

Aim 1, mother's perception of child's weight and actual weight classification, was tested, first by cross-tabulation and a significant positive relationship $(p<0.001)$ was found. Normal weight children, approximately $60 \%$ of the sample, were correctly classified by $57 \%$ of the mothers. However, despite the positive association, there were misclassification for over/obese children. For overweight and obese children, only $33.3 \%$ of the mothers classified their overweight or obese children correctly; while $67.7 \%$ perceived their children to be normal.

Aims 1 and 2, child's sex and mother's perception of child's BMI, were tested by a mixed linear model with dyads $(B=4.02$ $(2.84,5.68), p<0.001)$. Mothers perceive underweight $(\mathrm{B}=-6.66$ $(-9.76,-3.55), p<0.001)$ and normal weight $(B=-4.13(-7.11$, -1.15), $p=0.007$ ) children as having lower BMI as compared to overweight/obese children. The child's sex $(p=0.200)$ and the mother's perception by the child's sex $(p=0.203)$ were not significant indicators of actual BMI.

Aim 3 was tested by cross-tabulation. Mother's perception of child's weight was not significantly associated with her health belief that children need early weight management intervention ( $p=0.216$ ); however, there was a trend in this health belief and correct perception of child's weight $(p=0.072)$. Of the mothers who did not perceive their child's weight correctly, $58.6 \%$ agreed on the need for their children to have early weight management, while $41.4 \%$ responded that there should be no early weight management. Mothers who correctly perceived their child's weight $78.9 \%$ agree on the need for children to have early weight management while $21.1 \%$ did not think it was necessary to have early weight management.

\section{Discussion}

The current study demonstrated misclassification by mothers of their child's weight category. Although mothers perceived their underweight and normal weight children as having lower body weight as compared to mothers of overweight/obese children, approximately one-third underestimated their overweight/ obese child's weight. Over $40 \%$ of mother's who misclassified their child's weight did not think it was necessary to have early childhood weight management.
Sociodemographics and nationality of parents affect their perception of their child's weight and its implication for their physical health. Two-thirds of Australian mothers misclassified their children's body weight, but that most of the mothers $(83 \%)$ that recognized their child's weight issues had developed strategies managing their child's weight [10]. Parents from multiethnic mid-western schools who are overweight, have low income, and low education levels are less likely to note when their children become overweight; albeit, most of the mothers that had a family history of overweight were more likely to recognize whether children turned to be overweight [20].

In several studies of Western culture, parents who are overweight or have a family history of obesity are more likely to have plans to combat obesity with the exception of those with low income and education levels. This may not be the case in Kuwait. Nearly all (97\%) Kuwaiti mothers of overweight preschool children were unable to classify their child's weight [12]. Misclassification was more evident for male children and families without a history of obesity; however, mother's BMI, education level and child's age were not related to their perception [12]. The fact that there is a high prevalence of obesity in Kuwait may contribute to mothers' classifying their overweight child as 'normal'. Mothers in a high-obesity region had twice the odds of underestimating their overweight child compared to mothers in a low-obesity region in a national survey of Italian elementary school children [21]. Approximately twothirds of Emirati parents with overweight children misclassified their child's weight, which is essential for their participation in weight management programs [16]. Spargo \& Mellis [10] also support the need for parents to recognize weight issues in their child as crucial in designing weight management strategies. In a systematic review of primarily Western cultures, misperception of their overweight child was more likely for parents with younger children, ages 2-6 years as compared to parents with older, overweigh children [13]. In German population of mothers with children ages 3-6 years, approximately two-thirds of parents identified the overweight silhouette of a preschool child, of them, only half believed overweight preschoolers were at risk for chronic diseases [11]. Most of the German mothers indicated pediatric weight was related to genetic makeup rather than the eating behaviors [11]. In a global study of preschool obesity, increases in obesity were attributed to changes in eating behaviors and physical activity [9].

Our study shows that the child's BMI was associated with the mothers' perception of child's weight, regardless of the mother's BMI. Conversely, several studies reported that the male child's weight was underestimated more frequently than the female child's weight by their mothers for Kuwait, UK and Italian populations $[12,21,22]$. Kuwait mothers of preschool children who were younger and without a family history of obesity misperceive their children weight more often than older mothers; whereas, the child age, the mother's education, BMI, or working status were not determinants [12]. 
This study showed that there was a trend for the association of mother's health belief that her child needs early weight management and correct classification of her child's weight. This finding is promising since nearly $80 \%$ of mothers who correctly classified their child's weight thought it was necessary for their child to have early weight management. This health belief has not been reported in other Emirati populations, to date. Parent's perception of their child weight status was associated with their concern about their child's eating and activity habits in a U.S sample [23].

Our study has several strengths. It was the second study in Kuwait regarding mothers' perception of their children's BMI and extends the research to compare underweight, normal weight, and overweight/obese children with respect to mother's health perceptions and beliefs. The limitations of this study include small sample size which may have led to the failure to detect statistical difference with some variables or make it generalizable to Kuwaiti population. Another limitation was self-reported data that can lead to recall bias. Finally, this study did not include other family members, such as the fathers and the grandparents who may have an impact on the child's weight perception or the child's eating habits.

\section{Conclusion}

This study examined child's weight category and their mother's perception of their weight. In the present study, over $40 \%$ misclassified their child's weight and there were no differences by sex. This study assessed the health belief of mothers for their child's early weight management. Of the mothers who misclassified their child's weight, approximately $40 \%$ did not think early childhood weight management was necessary. Accurate identification of overweight and obesity in children by their mothers is very critical to prevent health consequences related to childhood obesity. Nutrition education and early childhood weight management programs have the potential-to prevention of childhood obesity and underweight in Kuwait; however, the health belief for early intervention is lacking. A change of attitude would be required for a child weight management program to be effective. The CDC chart for children and adolescents generates the sex and age specific BMI percentile. Adults were calculated using weight in $\mathrm{kg}$ and height in meters $\left(B M I=\mathrm{kg} / \mathrm{m}^{2}\right)$ using the CDC's standard for BMI classification, children and adults were classified as obese if their BMI was $\geq 95^{\text {th }}$ percentile, overweight if their BMI was between $\geq 85^{\text {th }}$ and $<95^{\text {th }}$, normal weight if their BMI was between $\geq 5^{\text {th }}$ and $<85^{\text {th }}$, and underweight if the BMI is $<5^{\text {th }}[18,19]$.

\section{References}

1. Kuwait population (2017) World population review.com.

2. (2006) Nutrition country profile: State of Kuwait.

3. Alsumaie M (2011) The Kuwait Nutrition Surveillance System (KNSS): an example for the region. Speech presented at Ibn Khaldoon Hall, Qatar.
4. Hudson C, Cherry DJ, Ratcliffe SJ, Mcclellan LC (2009) Head Start children's lifestyle behaviors, parental perceptions of weight, and body mass index. Journal of Pediatric Nursing 24(4): 292-301.

5. Lee JM, Pilli S, Gebremariam A, Keirns CC, Davis MM, et al. (2009) Getting heavier, younger: Trajectories of obesity over the life course. Int J Obes (Lond) 34(4): 614-623.

6. Vogels N, Posthumus DL, Mariman EC, Bouwman F, Kester AD, et al. (2006) Determinants of overweight in a Dutch children cohort. Am J Clin Nutr 84(4): 717-724.

7. Reilly JJ, Kelly J (2010) Long-term impact of overweight and obesity in childhood and adolescence on morbidity and premature mortality in adulthood: Systematic review. Int J Obes (Lond) 35(7): 891-898.

8. Musaad S, Paige K, Teran Garcia M, Donovan S, Fiese B, et al. (2013) Childhood overweight/obesity and pediatric asthma: The role of parental perception of child weight status. Nutrients 5(9): 3713-3729.

9. De Onis M, Blössner M, Borghi E (2010) Global prevalence and trends of overweight and obesity among preschool children. Am J Clin Nutr 92(5): 1257-1264.

10. Spargo M, Mellis C (2013) Childhood obesity and parental perceptions in a rural Australian population: A pilot study. J Paediatr Child Health 50(2): 131-134.

11. Warschburger P, Kroller K (2009) Maternal perception of weight status and health risks associated with obesity in children. Pediatrics 124(1): e60-e68.

12. Al Qaoud NM, Al Shami E, Prakash P (2010) Kuwaiti mothers' perception of their preschool children's weight status. J Dev Behav Pediatr 31(6): 505-510.

13. Rietmeijer Mentink M, Paulis WD, Middelkoop MV, Bindels PJ, Wouden JC (2012) Difference between parental perception and actual weight status of children: A systematic review. Matern Child Nutr 9(1): 3-22.

14. Maynard LM, Galuska DA, Blanck HM, Serdula MK (2003) Maternal perceptions of weight status of children. Pediatrics 111(5 Pt 2): 12261231.

15. Rosas LG, Harley KG, Guendelman S, Fernald, LCH, Mejia FF, et al. (2010) Maternal perception of child weight among Mexicans in California and Mexico. Matern Child Health J 14(6): 886-894.

16. Aljunaibi A, Abdulle A, Nagelkerke N (2013) Parental weight perceptions: a cause for concern in the prevention and management of childhood obesity in the United Arab Emirates. PLoS One 8(3): e59923.

17. (2011) Healthy weight-it's not a diet, it's a lifestyle!

18. Defining child obesity.

19. About Adult BMI. CDC.

20. Jaballas E, Clark Ott D, Clasen C, Stolfi A, Urban M (2011) Parents perceptions of their children's weight, eating habits, and physical activities at home and at school. J Pediatr Health Care 25(5): 294-301.

21. Binkin N, Spinelli A, Baglio G, Lamberti A (2013) What is common becomes normal: The effect of obesity prevalence on maternal perception. Nutr Metab Cardiovasc Dis 23(5): 410-416.

22. He M, Evans A (2007) Are parents aware that their children are overweight or obese? Can Fam Physician 53(9): 1493-1499.

23. Sylvetsky Meni AC, Gillepsie SE, Hardy T, Welsh JA (2015) The impact of parents' categorization of their own weight and their child's weight on healthy lifestyle promoting beliefs and practices. Journal of Obesity (2015): 1-7. 


\section{Current Research in Diabetes \& Obesity Journal} CC Commons Attribution 4.0 Licens

DOI: 10.19080/CRDOJ.2018.06.555693
Your next submission with Juniper Publishers will reach you the below assets

- Quality Editorial service

- Swift Peer Review

- Reprints availability

- E-prints Service

- Manuscript Podcast for convenient understanding

- Global attainment for your research

- Manuscript accessibility in different formats

( Pdf, E-pub, Full Text, Audio)

- Unceasing customer service

Track the below URL for one-step submission https://juniperpublishers.com/online-submission.php 